\title{
Increased plant sterol and stanol levels in brain of Watanabe rabbits fed rapeseed oil derived plant sterol or stanol esters
}

\author{
Christiane B. Fricke ${ }^{1}$, Malene Schrøder ${ }^{2}$, Morten Poulsen ${ }^{2}$, Klaus von Bergmann ${ }^{1}$, Ingmar Wester ${ }^{3}$, \\ Ib Knudsen $^{2}$, Alicja Mortensen ${ }^{2}$ and Dieter Lütjohann ${ }^{1 *}$ \\ ${ }^{1}$ Department of Clinical Pharmacology, University of Bonn, Sigmund-Freud-Strasse 25, D-53105 Bonn, Germany \\ ${ }^{2}$ Danish Institute for Food and Veterinary Research, Mфrkhфj Bygade 19, DK-2860 Sфborg Denmark \\ ${ }^{3}$ Raisio Group, PO Box 101, FI-21201 Raisio, Finland \\ (Received 10 November 2006 - Revised 23 March 2007 - Accepted 12 April 2007)
}

Foods containing plant sterol or stanol esters can be beneficial in lowering LDL-cholesterol concentration, a major risk factor for CVD. The present study examined whether high dietary intake of rapeseed oil (RSO) derived plant sterol and stanol esters is associated with increased levels of these components in brain tissue of homozygous and heterozygous Watanabe rabbits, an animal model for familial hypercholesterolemia. Homozygous animals received either a standard diet, RSO stanol or RSO sterol ester while heterozygous animals were additionally fed with $2 \mathrm{~g}$ cholesterol/kg to the respective diet form for $120 \mathrm{~d}$ ( $n 9$ for each group). Concentrations of cholesterol, its precursor lathosterol, plant sterols and stanols in brain and additionally in liver and plasma were determined by highly sensitive GC-MS. High-dose intake of RSO derived plant sterols and stanols resulted in increased levels of these components in plasma and liver. In brain a limited uptake of plant sterols and stanols was proven, indicating that these compounds passed the blood-brain barrier and may be retained in the brain tissue of Watanabe rabbits. Plant stanol ester feeding lowered plant sterol levels in brain, liver, and plasma. Cholesterol synthesis in brain, indicated by lathosterol, a local surrogate cholesterol synthesis marker, does not seem to be affected by plant sterol or stanol ester feeding. We conclude that high dose intake of plant sterol and stanol esters in Watanabe rabbits results in elevated concentrations of these components not only in the periphery but also in the central nervous system.

Phytosterols: Brain cholesterol: LDL receptor: Hypercholesterolemia: Atherosclerosis

Fortification of margarine and dairy products with plant sterol or plant stanol esters is a current dietary approach to lower elevated plasma cholesterol levels ${ }^{1-3}$. High levels of total and LDL cholesterol are risk factors for atherosclerosis and CHD, the major cause of morbidity and mortality in the Western world ${ }^{4}$. Watanabe heritable hyperlipidemic (Watanabe) rabbits, the animal model used in the present study, have a defect of the LDL receptor and consequently develop hypercholesterolemia, resulting in atherosclerotic lesions very similar to those in human subjects and xantomas ${ }^{5-7}$.

Comparable to human subjects the heterozygous form of hypercholesterolemia in Watanabe rabbits leads to a 2-3fold increase in plasma cholesterol, while the homozygous form presents up to 10-fold higher circulating plasma cholesterol levels ${ }^{6}$. The present study was part of the European Union project NOFORISK ('Quantitative risk assessment strategies for novel food') $)^{8}$. In this project, a scientific methodology necessary for quantitative risk assessment of 'second generation' novel foods to be marketed in the European Union was developed and validated. The primary goal of the Watanabe rabbit experiments within the project was to evaluate the presumable athero-protective or atherogenic character of plant sterol or stanol ester supplementation in cholesterol-fed heterozygous or chow-fed homozygous Watanabe rabbits. The present investigation examined, as a secondary goal, whether a high dietary intake of plant sterol or stanol esters is associated with increased levels of these components not only in the periphery (plasma and liver) but also in the central nervous system (brain) of heterozygous and homozygous Watanabe rabbits. It is generally assumed that cholesterol, plant sterols or stanols do not pass the bloodbrain barrier? ${ }^{9}$. However, the presence of the plant sterols campesterol and sitosterol have been reported in brain tissue of mice and $\operatorname{man}^{10-15}$, while so far, the presence of plant stanols, the $5 \alpha$-saturated metabolites of sterols, in brain tissue was only reported in a patient with sitosterolemia ${ }^{16}$. Furthermore, we wanted to investigate whether the possible presence of plant sterols and stanols in brain of the Watanabe rabbits could influence local cholesterol synthesis and content.

\section{Materials and methods}

Animals and diets

Twenty-seven homozygous and twenty-seven heterozygous Watanabe rabbits of both sexes, six-weeks old, were obtained 
from the in-house breeding colony of the Danish Institute for Food and Veterinary Research, Soeborg, Denmark (derived from a parent generation obtained from F.G.J. Janssen, University of Leiden, the Netherlands, with permission from Y. Watanabe). The animals were housed individually in stainless steel cages under controlled environmental conditions (temperature $18 \pm 2{ }^{\circ} \mathrm{C}$, relative humidity $55 \pm 5 \%, 12: 12 \mathrm{~h}$ light-dark cycle, air changed 10 times/h) and observed at least twice daily for any abnormalities in the clinical condition. Body weight was recorded weekly and feed intake daily. Rapeseed oil (RSO) sterols were sourced and either esterified as such with RSO fatty acids or first saturated to corresponding stanols and thereafter esterified with RSO fatty acids (Raisio Group, Raisio, Finland). The RSO sterol and stanol esters used in this study currently lack the required safety evaluation for their use as such in foods with added phytosterols. The homozygous Watanabe rabbits were randomised into three dietary groups receiving a standard diet (Altromin No. 2123; average content in the diet $(\mathrm{g} / \mathrm{kg})$ : crude protein 120 , crude fat 30 , crude fibre 200 , ash 90 , moisture 120 , nitrogen-free extract 440 , and metabolisable energy $963 \mathrm{~kJ} / 100 \mathrm{~g}$; Altromin International, Lage, Germany), standard diet with added RSO stanol ester $(34 \mathrm{~g} / \mathrm{kg}$, equivalent to $20 \mathrm{~g}$ plant stanols $/ \mathrm{kg}$ ) or standard diet with added RSO sterol ester $(34 \mathrm{~g} / \mathrm{kg}$, equivalent to $20 \mathrm{~g}$ plant sterols $/ \mathrm{kg})$, respectively (nine rabbits in each group; body weight: 0.96 (SD 0.07) kg). The heterozygous animals were randomised into three dietary groups receiving standard diet with added cholesterol $(2 \mathrm{~g} / \mathrm{kg})$, standard diet with added $2 \mathrm{~g}$ cholesterol $/ \mathrm{kg}$ and $34 \mathrm{~g} \mathrm{RSO}$ stanol esters $/ \mathrm{kg}$, or standard diet with added cholesterol $(2 \mathrm{~g} / \mathrm{kg}$ ) and $34 \mathrm{~g}$ RSO sterol esters $/ \mathrm{kg}$ (body weight: 1.05 (SD 0.040) kg). All animals were fed the specific diets $(100 \mathrm{~g} / \mathrm{d})$ for $120 \mathrm{~d}$ and had free access to tap water. In homozygous rabbits, the body weight increased during the experimental period feeding standard diet, or this diet with added stanol or sterol esters to 2623 (SD 140) g, 2626 (SD 178) g and 2632 (SD 111) g, respectively. In heterozygous rabbits, the body weight increased to 2884 (SD 176) g, 2732 (SD 124) g and 2837 (SD 171) g, respectively. The recommended maximal daily intake of phytosterols for human subjects in the European Union-market is $3 \mathrm{~g} / \mathrm{d}$ or $0.04 \mathrm{~g} / \mathrm{kg}$ body weight, equivalent to a twenty times lower dose than applied to the rabbits in the present study. These high doses of plant sterols and stanols were chosen to achieve measurable changes. Animal studies were performed under conditions approved by the Danish Agency for Protection of Experimental Animals and by the in-house Animal Welfare Committee.

\section{Plasma, tissue and feed sample preparation}

Blood samples were collected into tubes containing sodium heparin from the marginal ear vein of unanaesthetised animals after overnight fasting. The samples were taken prior to the first feeding of the respective diet (baseline) and at termination (endpoint). The blood was placed on a rotation bed and centrifuged at $2500 \mathrm{~g}$ for $15 \mathrm{~min}$. Plasma was immediately frozen and kept at $-20^{\circ} \mathrm{C}$ until analysed. The rabbits were euthanised by intravenous injection of pentobarbital $(100 \mathrm{mg} / \mathrm{kg}$ body weight) into the marginal ear vein, followed by exsanguination, and the central arterial system was perfused with about $300 \mathrm{ml}$ chilled $0.9 \% \mathrm{NaCl}$ solution through a canula, inserted into the left ventricle of the heart. From the rostral half of the right side of the brain and from the left lobe of the liver, aliquots were excised, weighed and kept frozen at $-20^{\circ} \mathrm{C}$. Prior to sterol analysis, brain and liver specimens were spun in a Speedvac AES 100 (Servant Instruments Inc., Farmingdale, NY, USA) at 12 mbar for $48 \mathrm{~h}$ until completely dried. Wet and dry weights were noted and sterols and stanols were extracted from the dried tissues in $2 \mathrm{ml}$ chloroform-methanol mixture $(2: 1 ; \mathrm{v} / \mathrm{v})$ for $48 \mathrm{~h}$. Feed was pulverised in a mortrar and $7 \mathrm{ml}$ chloroform-methanol mixture $(2: 1 ; \mathrm{v} / \mathrm{v})$ was added to the pulver $(30 \mathrm{mg})$ for extraction of sterols and stanols.

\section{Sterol extraction and determination}

$300 \mu \mathrm{l}$ brain and liver extracts and $2 \mathrm{ml}$ feed chloroformmethanol sterol extracts were evaporated to dryness under a stream of $\mathrm{N}_{2}$ at $63^{\circ} \mathrm{C}$. After alkaline hydrolysis the free sterols and stanols were extracted twice with $3 \mathrm{ml}$ cyclohexane and converted to trimethylsilylethers by adding $40 \mu$ l trimethylsilylation reagent (pyridine-hexamethyldisilazan-trimethylchlorosilane (9:3:1, by volume); Merck, Darmstadt, Germany). For determination of plasma sterol and stanol concentrations, sterols were extracted and derivatised from $50 \mu$ plasma as described $^{10}$. Feed was analysed in triplicate. Cholesterol concentrations were determined by GC-flame ionisation detection $^{17}$. Levels of plant sterols (campesterol, sitosterol, brassicasterol and stigmasterol), plant stanols (campestanol, sitostanol) and the cholesterol precursor lathosterol were determined using GC-MS with epicoprostanol (Sigma, Deisenhof, Germany) as internal standard ${ }^{17}$. The sterols and stanols were monitored as their trimethylsilyl derivates in the selected ion monitoring mode using the following masses: epicoprostanol m/z $370(\mathrm{M}+)$, lathosterol at m/z $458(\mathrm{M}+)$, campesterol at $\mathrm{m} / \mathrm{z} 472(\mathrm{M}+)$, sitosterol at $\mathrm{m} / \mathrm{z} 486$ $(\mathrm{M}+)$, stigmasterol at $\mathrm{m} / \mathrm{z} 484(\mathrm{M}+)$, brassicasterol at $\mathrm{m} / \mathrm{z}$ $470(\mathrm{M}+)$, campestanol at $\mathrm{m} / \mathrm{z} 474(\mathrm{M}+)$ and sitostanol at $\mathrm{m} / \mathrm{z} 488(\mathrm{M}+)$. Peak integration was performed manually and sterols were quantified from selected-ion monitoring analyses against internal standards using standard curves for the listed sterols. Identity of all sterols was proven by comparison with the full-scan mass spectra of authentic compounds.

\section{Statistical methods}

The results were expressed as means with their standard deviations. Differences for plasma parameters between baseline and endpoint were calculated by paired Student's two-tailed $t$ test using SPSS 12.0 for Windows (SPSS Inc., Chicago, IL, USA). Differences between the parameters for brain within the different groups were analysed by unpaired twotailed $t$ test. $P$ values $<0.05$ were considered significant.

\section{Results}

\section{Sterol and stanol concentration in feed}

Cholesterol and plant sterol/stanol concentrations in the experimental diets are given in Table 1 . Each diet given to the heterozygous rabbits contained about $2 \mu \mathrm{g}$ cholesterol/mg solid chow $(2 \mathrm{~g} / \mathrm{kg})$, while in each diet applied to the homozygous rabbits only a minor amount of cholesterol $(<0.03 \mu \mathrm{g} / \mathrm{mg}$ 
dry weight) could be detected. Percentage content of plant sterols and stanols in the different feed for heterozygous and homozygous rabbits, respectively, was comparable.

Plant sterol and stanol concentration in plasma, brain and liver

Heterozygous Watanabe rabbits. Feeding RSO plant stanol esters to heterozygous Watanabe rabbits resulted in a significantly higher total plant stanol concentration in plasma, brain, and liver compared to rabbits fed with cholesterol only (Tables 2 and 3). By contrast, total plant sterol concentrations in plasma, brain, and liver were lower in animals fed with RSO plant stanol esters, while total phytosterol levels (sum of plant sterols and plant stanols) were only lowered in the brains of animals of this group. In RSO sterol esterfed rabbits not only total plant sterol but also total plant stanol concentration in plasma, brain and liver tissues increased significantly compared to control. Therefore, total phytosterol level in RSO sterol ester-fed rabbits was about 7 times $(P<0.001)$ higher in plasma and brain and 13 times $(P<0.001)$ higher in liver compared with the rabbits fed with cholesterol only (Tables 2 and 3 ). Total plant stanol and sterol levels were significantly higher in the liver compared with brains from all animals (Fig. 1). Furthermore, in plasma from heterozygous Watanabe rabbits, the level of campesterol in the RSO sterol ester-fed group was about 25 times higher than the plasma level of campestanol in the RSO stanol ester-fed group. In brain, tissue level of campesterol in the RSO sterol ester-fed animals was about 50 times higher than the brain tissue level of campestanol in the RSO stanol ester-fed group while in liver it was about 24 times higher. The campesterol:sitosterol and campestanol:sitostanol ratios in plasma and brain are given in Table 4. In brain these ratios were significantly lower in all groups of heterozygous rabbits compared with the ratios in plasma.

Homozygous Watanabe rabbits. In homozygous rabbits, we also observed lower plasma, brain and liver total plant sterol and total phytosterol levels in the $34 \mathrm{~g} / \mathrm{kg}$ RSO stanol ester-fed rabbits compared with standard diet-fed animals while total plant stanol levels were higher (Tables 5 and 6). Moreover, as in heterozygous rabbits RSO plant sterol ester feeding increased total plant sterol and total plant stanol concentration in plasma, brain and liver. The concentrations of total phytosterols in RSO sterol ester-fed rabbits was about 4.4 times $(P<0.001)$ higher in plasma and brain and $5 \cdot 3$ times $(P<0.001)$ higher in liver compared with standard diet-fed rabbits (Tables 5 and 6). As already seen in heterozygous animals total plant stanol and sterol levels were significantly higher in the liver compared with brains from all animals (Fig. 2). In plasma of homozygous Watanabe rabbits, the level of campesterol in the RSO sterol ester-fed group was about 11 times higher than the plasma level of campestanol in the RSO stanol ester-fed rabbits. In brain tissue in the RSO sterol ester-fed rabbits campesterol concentration was 22 times and in the liver 17 times higher than campestanol in the RSO stanol ester-fed animals.

It was interesting that in the different groups of homozygous rabbits, plasma levels of plant sterols and plant stanols were approximately 5-15 times higher than in heterozygous rabbits, respectively, but concentrations of these substances 
Table 2. Cholesterol, lathosterol, lathosterol:cholesterol ratio and plant sterols and stanols in plasma before and during rapeseed oil derived plant sterol and plant stanol feeding with continuous cholesterol intake $(0.2 \mathrm{~g} / \mathrm{kg})$ for $120 \mathrm{~d}$ in heterozygous Watanabe rabbits

(Mean values with standard deviations for nine rabbits)

\begin{tabular}{|c|c|c|c|c|c|c|c|c|c|c|c|c|}
\hline \multirow[b]{3}{*}{ Plant sterol/stanol } & \multicolumn{4}{|c|}{ Cholesterol (2 g/kg) } & \multicolumn{4}{|c|}{ Cholesterol $(2 \mathrm{~g} / \mathrm{kg})+$ stanol ester $(34 \mathrm{~g} / \mathrm{kg})$} & \multicolumn{4}{|c|}{ Cholesterol $(2 \mathrm{~g} / \mathrm{kg})+$ sterol ester $(34 \mathrm{~g} / \mathrm{kg})$} \\
\hline & \multicolumn{2}{|c|}{ Baseline } & \multicolumn{2}{|c|}{ Endpoint } & \multicolumn{2}{|c|}{ Baseline } & \multicolumn{2}{|c|}{ Endpoint } & \multicolumn{2}{|c|}{ Baseline } & \multicolumn{2}{|c|}{ Endpoint } \\
\hline & Mean & SD & Mean & SD & Mean & SD & Mean & SD & Mean & SD & Mean & SD \\
\hline Cholesterol (mg/dl) & 107 & 29.6 & $490^{\star}$ & 370 & 139 & 33.1 & $158 \dagger$ & $85 \cdot 2$ & 123 & $36 \cdot 3$ & $177 \dagger$ & 115 \\
\hline Lathosterol (mg/dl) & 0.09 & 0.05 & 0.22 & 0.19 & 0.10 & 0.03 & $0.06^{\star} \dagger$ & 0.02 & 0.08 & 0.04 & 0.09 & 0.08 \\
\hline Lathosterol:Cholesterol $(\mu \mathrm{g} / \mathrm{mg})$ & 0.84 & 0.22 & $0.41^{* \star *}$ & $0 \cdot 12$ & 0.75 & $0 \cdot 14$ & $0.43^{\star \star \star}$ & $0 \cdot 15$ & 0.62 & 0.25 & $0.50^{\star}$ & 0.22 \\
\hline Campesterol (mg/dl) & $1 \cdot 38$ & 0.37 & $2 \cdot 22$ & 1.76 & 1.99 & 0.55 & $0.63^{\star \star \star} \dagger$ & 0.36 & 1.82 & 0.38 & $20 \cdot 3^{\star \star \star}+\dagger$ & $11 \cdot 7$ \\
\hline Sitosterol $(\mathrm{mg} / \mathrm{dl})$ & 0.42 & 0.15 & 0.65 & 0.35 & 0.57 & 0.18 & $0 \cdot 19^{\star \star \star} \dagger \dagger$ & 0.07 & 0.54 & 0.11 & $1.39^{\star *} \dagger$ & 0.72 \\
\hline Brassicasterol ( $\mu \mathrm{g} / \mathrm{dl})$ & $40 \cdot 2$ & $12 \cdot 7$ & $30 \cdot 8$ & $25 \cdot 6$ & $53 \cdot 8$ & $14 \cdot 3$ & $13 \cdot 9^{\star \star \star}$ & $3 \cdot 15$ & $42 \cdot 1$ & $9 \cdot 13$ & $142^{*} \dagger$ & 106 \\
\hline Stigmasterol $(\mu \mathrm{g} / \mathrm{dl})$ & 5.92 & 0.87 & $9 \cdot 78^{*}$ & $4 \cdot 14$ & 4.88 & $2 \cdot 17$ & $2 \cdot 52^{\star \star}+\dagger \dagger$ & 2.75 & $7 \cdot 24$ & 0.96 & $5 \cdot 14^{\star \star}+\dagger$ & 1.06 \\
\hline Total plant sterols (mg/dl) & 1.84 & 0.51 & 2.91 & $2 \cdot 13$ & $2 \cdot 62$ & 0.72 & $0.84^{\star \star *} \dagger$ & 0.42 & 2.41 & 0.47 & $21 \cdot 8^{\star \star \star}+\dagger \dagger$ & $12 \cdot 4$ \\
\hline Campestanol $(\mu \mathrm{g} / \mathrm{dl})$ & 31.8 & $6 \cdot 18$ & 68.0 & $47 \cdot 1$ & $42 \cdot 8$ & $15 \cdot 9$ & $817^{\star \star \star}+\dagger \dagger$ & 454 & $40 \cdot 3$ & 9.69 & $229^{\star \star \star}+\dagger \dagger$ & $93 \cdot 1$ \\
\hline Sitostanol $(\mu \mathrm{g} / \mathrm{dl})$ & $13 \cdot 0$ & $2 \cdot 83$ & $16 \cdot 0$ & 8.64 & $17 \cdot 4$ & $8 \cdot 17$ & $100^{\star \star}+\dagger$ & 59.8 & $16 \cdot 2$ & 2.91 & $25 \cdot 3$ & $15 \cdot 3$ \\
\hline Total plant stanols $(\mu \mathrm{g} / \mathrm{dl})$ & 44.8 & 8.84 & $84 \cdot 0^{\star}$ & 55.5 & $60 \cdot 1$ & $23 \cdot 6$ & $917^{\star \star \star}+\dagger \dagger$ & 513 & 56.5 & $12 \cdot 3$ & $254^{\star \star \star}+\dagger \dagger$ & 103 \\
\hline Total phytosterols $(\mathrm{mg} / \mathrm{dl}) \ddagger$ & 1.88 & 0.51 & 2.99 & $2 \cdot 19$ & $2 \cdot 68$ & 0.74 & $1 \cdot 75^{\star}$ & 0.93 & 2.47 & 0.48 & $22 \cdot 1^{\star \star \star}+\dagger \dagger$ & 12.5 \\
\hline
\end{tabular}

Mean values were significantly different at endpoint from baseline: ${ }^{*} P<0.05,{ }^{* \star} P<0.01,{ }^{* \star *} P<0.001$

Mean values were significantly different at endpoint $v$. endpoint cholesterol $(2 \mathrm{~g} / \mathrm{kg}): \dagger P<0.05,+\dagger P<0.01,+\dagger \dagger P<0.001$.

$\ddagger$ Sum of plant sterols and plant stanols.

Table 3. Cholesterol, lathosterol, the lathosterol:cholesterol ratio and plant sterols/stanols in brain and liver after rapeseed oil derived plant sterol and plant stanol feeding during continuous cholesterol intake $(2 \mathrm{~g} / \mathrm{kg})$ for $120 \mathrm{~d}$ in heterozygous Watanabe rabbits

(Mean values with standard deviations for nine rabbits)

\begin{tabular}{|c|c|c|c|c|c|c|c|c|c|c|c|c|}
\hline \multirow[b]{3}{*}{ Plant sterol/ stanol } & \multicolumn{7}{|c|}{ Brain } & \multicolumn{5}{|c|}{ Liver } \\
\hline & \multicolumn{2}{|c|}{$\begin{array}{l}\text { Cholesterol } \\
(2 \mathrm{~g} / \mathrm{kg})\end{array}$} & \multicolumn{2}{|c|}{$\begin{array}{l}\text { Cholesterol }(2 \mathrm{~g} / \mathrm{kg}) \\
+ \text { stanol ester }(34 \mathrm{~g} / \mathrm{kg})\end{array}$} & \multicolumn{2}{|c|}{$\begin{array}{c}\text { Cholesterol }(2 \mathrm{~g} / \mathrm{kg})+ \\
\text { sterol ester }(34 \mathrm{~g} / \mathrm{kg})\end{array}$} & \multicolumn{2}{|c|}{ Cholesterol (2 g/kg) } & \multicolumn{2}{|c|}{$\begin{array}{l}\text { Cholesterol }(2 \mathrm{~g} / \mathrm{kg})+ \\
\text { stanol ester }(34 \mathrm{~g} / \mathrm{kg})\end{array}$} & \multicolumn{2}{|c|}{$\begin{array}{r}\text { Cholesterol }(2 \mathrm{~g} / \mathrm{kg})+ \\
\text { sterol ester }(34 \mathrm{~g} / \mathrm{kg})\end{array}$} \\
\hline & Mean & SD & Mean & SD & Mean & SD & Mean & SD & Mean & SD & Mean & SD \\
\hline Cholesterol $(\mu \mathrm{g} / \mathrm{mg})$ & $70 \cdot 7$ & 8.24 & $63 \cdot 8^{*}$ & 4.66 & $59 \cdot 7^{\star}$ & 10.5 & $14 \cdot 0$ & 5.89 & $7 \cdot 35^{\star}$ & 3.02 & $6 \cdot 04^{\star *}$ & $2 \cdot 64$ \\
\hline Lathosterol $(\mu \mathrm{g} / \mathrm{mg})$ & 0.13 & 0.02 & $0 \cdot 10^{\star \star}$ & 0.01 & 0.11 & 0.02 & 0.005 & 0.002 & 0.005 & 0.004 & 0.008 & 0.010 \\
\hline Lathosterol:Cholesterol (ng/mg) & 1.78 & 0.23 & 1.61 & 0.14 & 1.82 & 0.20 & 0.37 & 0.20 & 0.83 & 0.65 & 1.71 & 2.82 \\
\hline Campesterol (ng/mg) & $17 \cdot 1$ & 3.69 & $7 \cdot 78^{\star \star \star}$ & $2 \cdot 22$ & $200^{\star \star \star}$ & $49 \cdot 6$ & $50 \cdot 4$ & $19 \cdot 7$ & $26 \cdot 4^{\star \star}$ & $12 \cdot 2$ & $822^{\star \star \star}$ & 272 \\
\hline Sitosterol (ng/mg) & 11.9 & 2.07 & $6 \cdot 13^{\star \star \star}$ & 0.69 & $19 \cdot 3^{\star \star \star}$ & 4.07 & $14 \cdot 8$ & 5.43 & $6 \cdot 00^{\star \star \star}$ & 3.87 & $47 \cdot 1^{\star \star \star}$ & $17 \cdot 4$ \\
\hline Brassicasterol (ng/mg) & 1.51 & 0.20 & $1 \cdot 13^{* \star *}$ & 0.22 & $4 \cdot 04^{\star \star *}$ & 1.06 & 1.04 & 0.67 & 0.54 & 0.28 & $6 \cdot 19^{\star \star \star}$ & $2 \cdot 80$ \\
\hline Stigmasterol (ng/mg) & 0.67 & 0.14 & $0.45^{\star \star \star}$ & 0.08 & 0.57 & 0.16 & 0.21 & 0.12 & 0.14 & 0.06 & 0.20 & 0.06 \\
\hline Total plant sterols (ng/mg) & $31 \cdot 2$ & 5.94 & $15 \cdot 5^{\star \star *}$ & 2.62 & $224^{\star \star *}$ & 53.9 & 66.5 & 23.5 & $33 \cdot 3^{\star *}$ & $15 \cdot 7$ & $876^{\star \star \star}$ & 285 \\
\hline Campestanol (ng/mg) & 0.71 & 0.14 & $4 \cdot 00^{\star \star \star}$ & 0.87 & 3.00 & 0.67 & 1.42 & 0.44 & $34 \cdot 1^{\star \star \star}$ & $18 \cdot 1$ & $8 \cdot 36^{\star \star \star}$ & 3.45 \\
\hline Sitostanol (ng/mg) & 0.45 & $0 \cdot 12$ & $0.81^{\star \star *}$ & 0.24 & $0 \cdot 60^{\star}$ & $0 \cdot 13$ & 0.45 & 0.13 & $2 \cdot 79^{\star \star \star}$ & 1.32 & 0.55 & 0.27 \\
\hline Total plant stanols (ng/mg) & $1 \cdot 16$ & 0.23 & $4 \cdot 81^{\star \star *}$ & 1.01 & $3 \cdot 60^{\star * \star}$ & 0.77 & 1.87 & 0.56 & $36 \cdot 9^{\star \star \star}$ & $19 \cdot 4$ & $8 \cdot 91^{\star \star \star}$ & $3 \cdot 71$ \\
\hline Total phytosterols $(\mathrm{ng} / \mathrm{mg}) \dagger$ & $32 \cdot 4$ & $6 \cdot 1$ & $20 \cdot 3^{\star \star \star}$ & 3.03 & $228^{\star \star \star}$ & 54.5 & $68 \cdot 6$ & $24 \cdot 1$ & $70 \cdot 2$ & $33 \cdot 7$ & $885^{\star \star \star}$ & 289 \\
\hline
\end{tabular}

Mean values were significantly different from those for cholesterol $(2 \mathrm{~g} / \mathrm{kg}):{ }^{\star} P<0.05,{ }^{\star \star} P<0.01,{ }^{\star \star \star} P<0.001$

† Sum of plant sterols and plant stanols 
(A)

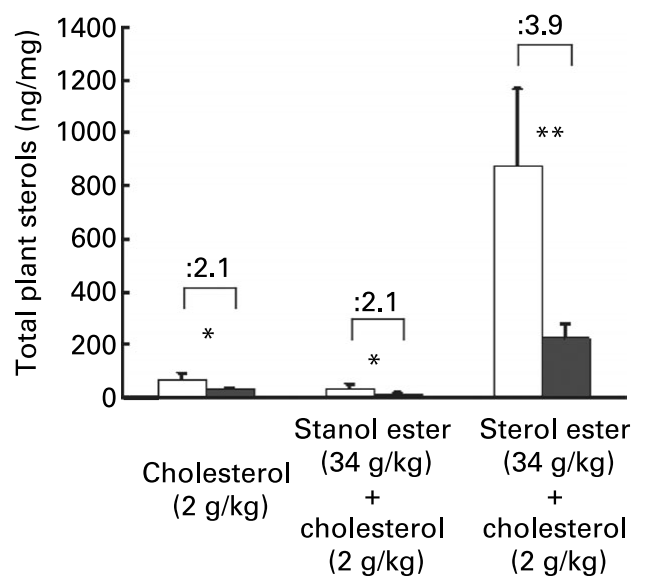

(B)

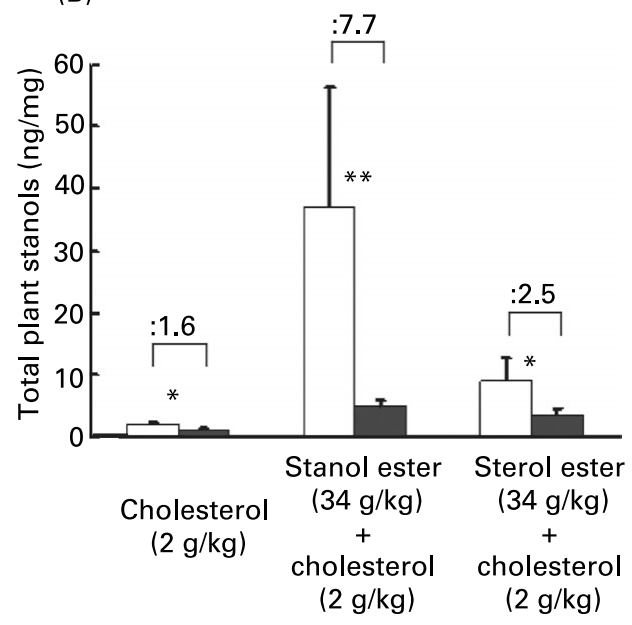

Fig. 1. Total plant sterol (A) and stanol (B) concentrations in brain ( $\square$ ) and liver ( $\square$ ) after rapeseed oil derived plant sterol and plant stanol feeding for 120 d in heterozygous Watanabe rabbits $(n 9)$. Values are means with standard deviations shown by vertical bars. Mean values were significantly different between brain and liver: ${ }^{\star} P<0.01$, ${ }^{\star \star} P<0.001$. Values above columns represent the quotient between liver and brain concentrations.

in brain were only 1-2 times higher. The campesterol:sitosterol and campestanol:sitostanol ratios in brains of homozygous animals was significantly lower compared with the ratios in plasma (Table 4).

\section{Cholesterol, lathosterol and the lathosterol: cholesterol ratio} in plasma, brain, and liver

Heterozygous Watanabe rabbits. Heterozygous rabbits fed for $120 \mathrm{~d}$ with cholesterol $(2 \mathrm{~g} / \mathrm{kg})$ only, showed on average a 3.6-fold $(P=0.013)$ increase in their plasma cholesterol level while pre-feeding (baseline) and terminal (endpoint) plasma cholesterol levels were not significantly different in the animals additionally fed plant stanol or sterol esters (Table 2). Feeding the rabbits with cholesterol $(2 \mathrm{~g} / \mathrm{kg})$ and RSO sterol or stanol esters for 120 d resulted in lower cholesterol concentrations in plasma, brain, and liver of the animals compared with rabbits fed with cholesterol only (Tables 2 and 3). In plasma the lathosterol:cholesterol ratio, indicator of endogenous cholesterol synthesis, decreased in all groups during the feeding period for $120 \mathrm{~d}$. Differences in endpoint lathosterol:cholesterol ratios between the groups, in plasma as well as in brain and liver were not detected. Absolute lathosterol concentration was only lower in plasma and brain of the rabbits fed with $2 \mathrm{~g}$ cholesterol/ $\mathrm{kg}$ and RSO stanol esters compared with the rabbits fed with cholesterol only (Tables 2 and 3). Significantly higher cholesterol levels were only found in female (258 (SD 60.5) $\mathrm{mg} / \mathrm{dl})$ compared with male $(108$ (SD $32 \cdot 3) \mathrm{mg} / \mathrm{dl}, P=0.001)$ heterozygous rabbits fed with cholesterol and plant stanol ester after $120 \mathrm{~d}$. However, baseline $v$. endpoint comparison of cholesterol in female and male animals of this feeding group revealed no significant difference (data not shown). Thus, the data for cholesterol in male and female rabbits were merged.

Homozygous Watanabe rabbits. In homozygous rabbits, no significant differences were found in the pre-feeding and terminal plasma cholesterol level in any of the three groups (Table 5). Furthermore, no differences in plasma, brain, and liver cholesterol level between the groups could be detected (Tables 5 and 6). As seen in plasma of heterozygous rabbits, feeding with different diets for $120 \mathrm{~d}$ resulted in a decrease of the lathosterol:cholesterol ratio in all groups. Differences

Table 4. Campesterol: sitosterol and campestanol:sitostanol ratios in plasma and brain after rapeseed oil (RSO) derived plant sterol and plant stanol ester feeding for $120 \mathrm{~d}$ in heterozygous and homozygous Watanabe rabbits

(Mean values with standard deviations for nine rabbits)

\begin{tabular}{|c|c|c|c|c|c|c|c|c|}
\hline \multirow[b]{3}{*}{ Feed } & \multicolumn{4}{|c|}{ Campesterol:sitosterol } & \multicolumn{4}{|c|}{ Campestanol:sitostanol } \\
\hline & \multicolumn{2}{|c|}{ Plasma } & \multicolumn{2}{|c|}{ Brain } & \multicolumn{2}{|c|}{ Plasma } & \multicolumn{2}{|c|}{ Brain } \\
\hline & Mean & SD & Mean & SD & Mean & SD & Mean & SD \\
\hline \multicolumn{9}{|l|}{ Heterozygous rabbits } \\
\hline Cholesterol $(2 \mathrm{~g} / \mathrm{kg})$ & 2.99 & $1 \cdot 14$ & $1 \cdot 41^{\star *}$ & 0.14 & 3.98 & 0.82 & $1 \cdot 65^{\star \star \star}$ & 0.40 \\
\hline Cholesterol $(2 \mathrm{~g} / \mathrm{kg})+$ RSO stanol ester $(34 \mathrm{~g} / \mathrm{kg})$ & $3 \cdot 28$ & 1.06 & $1 \cdot 26^{\star \star \star}$ & 0.31 & $8 \cdot 33$ & 0.85 & $5 \cdot 10^{\star \star \star}$ & $1 \cdot 13$ \\
\hline Cholesterol $(2 \mathrm{~g} / \mathrm{kg})+$ RSO sterol ester $(34 \mathrm{~g} / \mathrm{kg})$ & $15 \cdot 2$ & $5 \cdot 44$ & $10 \cdot 4^{\star}$ & 1.33 & $10 \cdot 4$ & 3.58 & $5 \cdot 04^{\star \star}$ & 0.63 \\
\hline \multicolumn{9}{|l|}{ Homozygous rabbits } \\
\hline Standard diet & $2 \cdot 28$ & 0.33 & $2 \cdot 02^{*}$ & 0.22 & $3 \cdot 28$ & 0.58 & $1 \cdot 42^{\star \star \star}$ & 0.25 \\
\hline RSO stanol ester (34 g/kg) & 2.71 & 0.40 & $1 \cdot 31^{\star \star \star}$ & 0.40 & $7 \cdot 73$ & $1 \cdot 21$ & $4 \cdot 82^{\star \star \star}$ & 0.91 \\
\hline RSO sterol ester $(34 \mathrm{~g} / \mathrm{kg})$ & $10 \cdot 0$ & $1 \cdot 29$ & $9 \cdot 58$ & 0.97 & $8 \cdot 33$ & $2 \cdot 32$ & $3 \cdot 21^{\star \star \star}$ & 0.63 \\
\hline
\end{tabular}

Mean values were significantly different between brain and plasma: ${ }^{\star} P<0.05,{ }^{\star \star} P<0.01,{ }^{\star \star \star} P<0.001$ 
Table 5. Cholesterol, lathosterol, the lathosterol:cholesterol ratio and plant sterols and stanols in plasma before and during rapeseed oil derived plant sterol and plant stanol feeding for $120 \mathrm{~d}$ in homozygous Watanabe rabbits

(Mean values with standard deviations for nine rabbits)

\begin{tabular}{|c|c|c|c|c|c|c|c|c|c|c|c|c|}
\hline \multirow[b]{3}{*}{ Plant sterol/stanol } & \multicolumn{4}{|c|}{ Standard diet } & \multicolumn{4}{|c|}{ Stanol ester (34 g/kg) } & \multicolumn{4}{|c|}{ Sterol ester (34 g/kg) } \\
\hline & \multicolumn{2}{|c|}{ Baseline } & \multicolumn{2}{|c|}{ Endpoint } & \multicolumn{2}{|c|}{ Baseline } & \multicolumn{2}{|c|}{ Endpoint } & \multicolumn{2}{|c|}{ Baseline } & \multicolumn{2}{|c|}{ Endpoint } \\
\hline & Mean & SD & Mean & SD & Mean & SD & Mean & SD & Mean & SD & Mean & SD \\
\hline Cholesterol (mg/dl) & 960 & 133 & 966 & 139 & 925 & 243 & 1016 & 271 & 951 & 175 & 884 & 146 \\
\hline Lathosterol (mg/dl) & 1.07 & 0.30 & $0.63^{\star \star \star}$ & 0.31 & 1.32 & 0.47 & $0.62^{\star \star \star}$ & 0.21 & $1 \cdot 16$ & 0.27 & $0.68^{\star \star \star}$ & 0.09 \\
\hline Lathosterol:Cholesterol $(\mu \mathrm{g} / \mathrm{mg})$ & $1 \cdot 13$ & 0.35 & $0.66^{\star \star}$ & 0.26 & 1.46 & 0.45 & $0.67^{\star \star \star}$ & 0.31 & 1.23 & 0.28 & $0.78^{\star \star \star}$ & 0.10 \\
\hline Campesterol (mg/dl) & 11.9 & $2 \cdot 17$ & $16 \cdot 5^{\star \star \star}$ & $2 \cdot 67$ & 11.7 & 1.61 & $6 \cdot 37^{\star \star \star}+\dagger \dagger$ & 1.50 & $10 \cdot 4$ & 1.34 & $99 \cdot 5^{\star \star *}+\dagger \dagger$ & 18.6 \\
\hline Sitosterol (mg/dl) & $5 \cdot 18$ & 1.06 & $7 \cdot 30^{\star *}$ & 1.13 & 4.80 & 0.73 & $2 \cdot 40^{\star \star \star}+\dagger \dagger$ & 0.62 & 3.49 & 0.70 & 9.95 $5^{\star \star \star}+† \dagger$ & 1.60 \\
\hline Brassicasterol ( $\mu \mathrm{g} / \mathrm{dl})$ & 323 & $56 \cdot 0$ & $211^{\star \star}$ & 111 & 334 & 44.5 & $67 \cdot 6^{\star \star *}+1$ & 30.8 & 356 & $54 \cdot 0$ & $1022^{\star \star *}+\dagger \dagger$ & 210 \\
\hline Stigmasterol $(\mu \mathrm{g} / \mathrm{dl})$ & 44.0 & 14.7 & $64 \cdot 3^{\star \star}$ & 9.04 & 41.6 & $12 \cdot 2$ & $15 \cdot 7^{\star \star \star}+\dagger \dagger$ & 4.02 & 31.6 & $10 \cdot 6$ & $12 \cdot 6^{\star \star \star}+\dagger \dagger$ & 0.96 \\
\hline Total plant sterols (mg/dl) & 17.4 & 3.23 & $24 \cdot 1^{\star \star \star}$ & 3.53 & $16 \cdot 9$ & $2 \cdot 24$ & $8 \cdot 85^{\star * \star}+\dagger \dagger$ & 2.06 & $14 \cdot 3$ & $2 \cdot 07$ & $111^{\star \star *}+\dagger \dagger$ & $19 \cdot 8$ \\
\hline Campestanol $(\mu \mathrm{g} / \mathrm{dl})$ & 391 & $74 \cdot 2$ & $780^{\star \star \star}$ & 154 & 313 & 124 & $8657^{\star \star \star}+† \dagger$ & 1879 & 283 & $56 \cdot 9$ & $1141^{* *+\dagger}$ & 265 \\
\hline Sitostanol $(\mu \mathrm{g} / \mathrm{dl})$ & 188 & 33.9 & $243^{*}$ & $52 \cdot 2$ & 167 & 82.4 & $1129^{\star \star \star}+\dagger \dagger$ & 250 & 140 & 26.5 & $140+† \dagger$ & $22 \cdot 0$ \\
\hline Total plant stanols $(\mu \mathrm{g} / \mathrm{dl})$ & 579 & 96.4 & $1023^{\star \star \star}$ & 189 & 480 & 190 & $9787^{\star \star \star}+\dagger \dagger$ & 2076 & 423 & $82 \cdot 1$ & $1281^{i \neq+t}$ & 261 \\
\hline Total phytosterols $(\mathrm{mg} / \mathrm{dl}) \ddagger$ & $18 \cdot 0$ & $3 \cdot 24$ & $25 \cdot 1^{\star \star *}$ & 3.63 & $17 \cdot 3$ & $2 \cdot 27$ & $18 \cdot 7 \dagger \dagger$ & 3.80 & $14 \cdot 7$ & $2 \cdot 14$ & $112^{\star \star \star}+† \dagger$ & $20 \cdot 0$ \\
\hline
\end{tabular}

Mean values were significantly different at endpoint $v$. baseline: ${ }^{*} P<0.05,{ }^{* *} P<0.01,{ }^{* \star *} P<0.001$

Mean values were significantly different at endpoint $v$. endpoint Standard diet: $t P<0.05,+t P<0.01, \mathrm{tt}+P<0.001$

$\ddagger$ Sum of plant sterols and plant stanols.

Table 6. Cholesterol, lathosterol, the lathosterol:cholesterol ratio and plant sterols/stanols in brain and liver after rapeseed oil derived plant sterol and plant stanol feeding for $120 \mathrm{~d}$ in homozygous Watanabe rabbits

(Mean values with standard deviations for nine rabbits)

\begin{tabular}{|c|c|c|c|c|c|c|c|c|c|c|c|c|}
\hline \multirow[b]{3}{*}{ Plant sterol/ stanol } & \multicolumn{6}{|c|}{ Brain } & \multicolumn{6}{|c|}{ Liver } \\
\hline & \multicolumn{2}{|c|}{ Standard diet } & \multicolumn{2}{|c|}{$\begin{array}{l}\text { Stanol ester } \\
(34 \mathrm{~g} / \mathrm{kg})\end{array}$} & \multicolumn{2}{|c|}{ Sterol ester (34 g/kg) } & \multicolumn{2}{|c|}{ Standard diet } & \multicolumn{2}{|c|}{ Stanol ester $(34 \mathrm{~g} / \mathrm{kg})$} & \multicolumn{2}{|c|}{ Sterol ester $(34 \mathrm{~g} / \mathrm{kg})$} \\
\hline & Mean & SD & Mean & SD & Mean & SD & Mean & SD & Mean & SD & Mean & SD \\
\hline Cholesterol $(\mu \mathrm{g} / \mathrm{mg})$ & 60.5 & 10.5 & $70 \cdot 3$ & $9 \cdot 39$ & $62 \cdot 8$ & $10 \cdot 1$ & 7.66 & 1.32 & $8 \cdot 61$ & 0.97 & $7 \cdot 25$ & 1.48 \\
\hline Lathosterol ( $\mu \mathrm{g} / \mathrm{mg})$ & 0.11 & 0.03 & 0.13 & 0.02 & 0.11 & 0.02 & 0.014 & 0.007 & 0.022 & 0.014 & 0.016 & 0.010 \\
\hline Lathosterol:Cholesterol (ng/mg) & 1.89 & 0.21 & 1.79 & 0.22 & 1.82 & 0.12 & $1 \cdot 81$ & 0.86 & $2 \cdot 62$ & $1 \cdot 81$ & $2 \cdot 26$ & 1.59 \\
\hline Campesterol (ng/mg) & $32 \cdot 2$ & $9 \cdot 27$ & $15 \cdot 8^{\star \star \star}$ & $5 \cdot 61$ & $198^{\star \star \star}$ & $42 \cdot 0$ & 163 & $32 \cdot 0$ & $63 \cdot 1^{\star \star \star}$ & $18 \cdot 0$ & $1102^{\star \star \star}$ & 376 \\
\hline Sitosterol (ng/mg) & $16 \cdot 0$ & 3.24 & $11 \cdot 8^{\star *}$ & 1.92 & $20 \cdot 6^{\star}$ & 3.64 & $56 \cdot 4$ & 14.5 & $23 \cdot 0^{\star \star \star}$ & 7.91 & $102^{* *}$ & $32 \cdot 8$ \\
\hline Brassicasterol (ng/mg) & 1.95 & 0.53 & $1.46^{\star}$ & 0.21 & $3 \cdot 80^{\star \star \star}$ & 0.72 & 3.67 & 1.09 & $2 \cdot 15^{\star \star}$ & 0.73 & $11.5^{\star \star \star}$ & 3.98 \\
\hline Stigmasterol (ng/mg) & 0.86 & 0.18 & 0.79 & 0.23 & 0.73 & 0.19 & 1.75 & 0.16 & $1 \cdot 12^{\star \star \star}$ & 0.41 & $0.90^{\star \star *}$ & 0.21 \\
\hline Total plant sterols $(\mathrm{ng} / \mathrm{mg})$ & 51.0 & $12 \cdot 9$ & $29 \cdot 8^{\star \star \star}$ & 6.63 & $223^{\star \star \star}$ & $45 \cdot 6$ & 225 & 42.9 & $89 \cdot 4^{\star \star \star}$ & $25 \cdot 8$ & $1216^{\star \star \star}$ & 408 \\
\hline Campestanol (ng/mg) & 0.96 & 0.18 & $8 \cdot 82^{\star \star \star}$ & 1.93 & $2 \cdot 36^{\star \star \star}$ & 0.56 & $4 \cdot 27$ & $1 \cdot 32$ & $66 \cdot 5^{\star \star \star}$ & $25 \cdot 2$ & $11 \cdot 8^{\star \star}$ & 5.78 \\
\hline Sitostanol (ng/mg) & 0.69 & $0 \cdot 16$ & $1.85^{\star \star \star}$ & 0.35 & 0.73 & $0 \cdot 11$ & 1.41 & 0.64 & $7 \cdot 77^{\star \star *}$ & $2 \cdot 27$ & $1 \cdot 20$ & 0.34 \\
\hline Total plant stanols (ng/mg) & 1.66 & 0.31 & $10 \cdot 7^{\star \star \star}$ & $2 \cdot 14$ & $3 \cdot 09^{\star \star \star}$ & 0.64 & 5.67 & 1.89 & $74 \cdot 3^{\star \star \star}$ & $27 \cdot 4$ & $13 \cdot 0^{\star \star}$ & 6.08 \\
\hline Total phytosterols $(\mathrm{ng} / \mathrm{mg}) \dagger$ & 52.6 & $13 \cdot 1$ & $40 \cdot 6^{\star}$ & 8.64 & $226^{\star \star \star}$ & $46 \cdot 1$ & 231 & 44.3 & $164^{\star \star}$ & $50 \cdot 3$ & $1229^{\star \star \star}$ & 414 \\
\hline
\end{tabular}

Mean values were significantly different from those for the standard diet: ${ }^{\star} P<0.05,{ }^{* \star} P<0.01,{ }^{* \star \star} P<0.001$.

† Sum of plant sterols and plant stanols. 
(A)

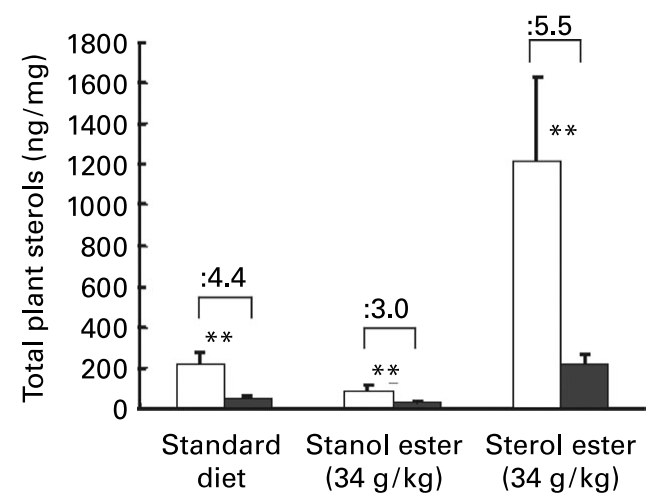

(B)

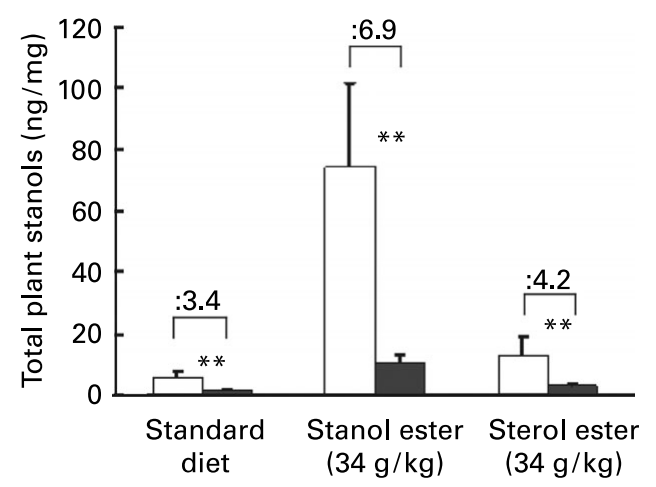

Fig. 2. Total plant sterol (A) and stanol (B) concentrations in brain ( $\square$ ) and liver ( $\square$ ) after rapeseed oil derived plant sterol and plant stanol feeding for $120 \mathrm{~d}$ in homozygous Watanabe rabbits $(n 9)$. Values are means with standard deviations shown by vertical bars. Mean values were significantly different between brain and liver: ${ }^{* \star} P<0.001$. Values above columns represent the quotient between liver and brain concentrations.

between the groups in plasma as well as in brain and liver were not observed (Tables 5 and 6). Independent of the diet, baseline lathosterol concentrations in plasma were higher in all homozygous rabbits compared with endpoint lathosterol concentrations (Table 5). Differences in lathosterol levels in plasma, brain, and liver between the dietary groups were not proven. As in heterozygous rabbits, significantly higher cholesterol levels in female (1222 (SD 131) mg/dl) compared with male (758 (SD 113) mg/dl, $P=0.001)$ homozygous rabbits were found in the stanol ester-fed group after $120 \mathrm{~d}$. Since baseline $v$. endpoint comparison of cholesterol in female and male animals of this feeding group also revealed no significant difference (data not shown), the data for cholesterol in male and female homozygous rabbits were merged. In homozygous Watanabe rabbits, pre-feeding cholesterol levels in plasma were approximately up to 10 times higher than in heterozygous rabbits. Although in homozygous Watanabe rabbits cholesterol levels in plasma were much higher, cholesterol concentrations in their brains did not differ from those found in heterozygous rabbits.

\section{Discussion}

Feeding a diet fortified with RSO plant sterol or stanol esters to heterozygous and homozygous Watanabe rabbits, resulted in increased levels of plant sterols and stanols not only in peripheral compartments (plasma and liver) but also in the brain. Consequently, plant sterols and stanols seem to be able to cross the blood-brain barrier. Increase in plasma, brain and hepatic plant stanol concentrations in RSO sterol ester-fed rabbits indicated that plant sterols are converted to plant stanols within the mammalian body. Application of plant stanol esters increased plant stanol levels while plant sterol levels decreased in plasma, brain and liver. In homozygous Watanabe rabbits, RSO plant sterols and stanols had no influence on plasma, brain and liver cholesterol. In contrast, in cholesterol-fed heterozygous rabbits, feeding a diet with added RSO plant sterols or stanols decreased cholesterol levels in plasma, brain and liver when compared to animals receiving the diet with only $2 \mathrm{~g}$ cholesterol $/ \mathrm{kg}$ added. In plasma of non-cholesterol-fed homozygous and cholesterol-fed heterozygous rabbits the lathosterol:cholesterol ratio, indicator of endogenous cholesterol synthesis, decreased in all groups during the feeding period for $120 \mathrm{~d}$ while no differences in endpoint lathosterol:cholesterol ratios between the groups, in plasma as well as in brain and liver, were detected.

In the brains of non-sitosterolemic human subjects and wild-type animals low concentrations of plant sterols, ranging from $10-80 \mathrm{ng} / \mathrm{mg}$, have been described ${ }^{10,11,14,18-21}$. In the brain of a patient with sitosterolemia, a rare, inherited sterol storage disease with a defect in the gene(s) for ABCG5/G8 transporters $^{22,23}$, campesterol $(200 \mathrm{ng} / \mathrm{mg})$ was found in concentrations comparable to sterol ester-fed rabbits of the present study ${ }^{16}$. ABCG5 and ABCG8 are half transporters which are expressed in the intestine and the liver where they re-secrete plant sterols and stanols back from the enterocytes into the lumen of the intestine, or mediate efflux of phytosterols and stanols as well as cholesterol from hepatocytes into the bile $\mathrm{e}^{4,24}$. Plasma levels of plant sterols in sitosterolemic patients $^{16,25}$ and in the rabbits used in the present study were also comparable. The only difference between our model and patients with sitosterolemia was that these patients had higher concentrations of sitosterol than campesterol in plasma and tissues. Recent studies demonstrated a 7- to 20fold increase in brain plant sterol levels in chow-fed ABCG5/G8 deficient mice ${ }^{11,12}$ and an increase of about $30 \%$ in plant sterol-fed $(8.3 \mathrm{~g} / \mathrm{kg})$ wild type mice ${ }^{12}$.

There are a great number of animal studies using plant stanol and sterol ester fortified food; however, only data on serum and liver but not on brain are available. In the present study, it was demonstrated that an increase in plasma plant sterol and stanol levels may result in increased levels of plant sterols and stanols in liver and in the brain. However, in healthy human subjects serum levels of phytosterols are typically much lower ${ }^{26-29}$ than those obtained in the present study with Watanabe rabbits. As a consequence, brain tissue levels of this component are assumed to be also lower. From previous studies it is well established that patients homozygous and heterozygous for familial hypercholesterolemia not only present increased cholesterol but also plant sterol levels. A plant sterol rich diet in combination with statins in these patients results in even more elevated serum plant sterol levels ${ }^{30}$. Whether or not this therapeutic approach in these patients has an effect on their plant sterol level in 
brain needs to be investigated, possibly in combination with neurological examination using cerebrospinal fluids.

Our findings provide further evidence that not only plant sterols but also plant stanols can cross the blood-brain barrier or that they are formed locally from sterols. Increased amounts of the plant stanols, campestanol and sitostanol, were also found in plasma, brain and liver in the animals fed with RSO sterol esters. Presumably, these stanols could have been produced endogenously from their unsaturated precursors campesterol and sitosterol in the mammalian body, also demonstrated by a previous study in subjects with sitosterolemia $^{15}$. In fact, feed enriched with $34 \mathrm{~g}$ RSO sterol esters $/ \mathrm{kg}$ also contained small amounts of plant stanols, probably formed during the production process. However, percentage distribution of campestanol and sitostanol in plasma (approximately $90 \%$ and $10 \%$, respectively) and brain (80\% and $20 \%$, respectively) compared with feed $(50 \%$ and $50 \%$, respectively) indicates that the shift is comparable to that of campesterol to sitosterol (about $90 \%$ and $10 \%$ in brain and plasma, respectively).

The question remains as to how plant sterols and stanols are transported into the brain. In a previous study, chow-fed ABCG5 and ABCG8-deficient and plant sterol-fed $(8.3 \mathrm{~g} / \mathrm{kg})$ wild type mice showed significantly elevated plant sterol levels, especially of campesterol, in the brain, while in ApoE-deficient mice they were not increased ${ }^{12}$. Watanabe rabbits show a 4- to 7-fold increase in plasma levels of $\mathrm{ApoE}^{31}$. Therefore, the possibility remains that $\mathrm{ApoE}$ is involved in this transport. ABCG5/8 transporter expression at the level of the blood-brain barrier is extremely low and it appears very unlikely that they play a role in plant sterol transport ${ }^{12}$.

It is noteworthy, that despite the approximately 10 -fold higher plasma and liver plant sterol and stanol levels in homozygous Watanabe rabbits compared to heterozygous Watanabe rabbits, the concentrations of plant sterols and stanols in the brain of these animals did not reveal such differences. This finding indicates a limit for sterol and/or for stanol uptake into the brain. Independent from the genotype and diet of the Watanabe animals, the plant sterol and stanol levels in the liver are significanly higher than in the brain (Figs. 1 and 2). Thus, the blood-brain barrier seems to protect the brain from excessive uptake of circulating phytosterols.

The campesterol:sitosterol ratios in plasma and brain were different (Table 4). Therefore, it seems obvious that high levels of non-cholesterol sterols in brain are not attributed to contaminations from the circulation by microvessels. In agreement with a previous study ${ }^{32}$, campesterol uptake within the intestine was presumably higher than that of sitosterol, indicated by higher plasma campesterol concentrations. By contrast, the lower campesterol:sitosterol and campestanol: sitostanol ratios in brain compared to these ratios in plasma reflected that sitosterol and sitostanol seem to pass the blood-brain barrier easier than campesterol and campestanol. Either there was relatively more sitosterol and sitostanol taken up into the brain or the excretion of campesterol and campestanol from the brain was higher than that for sitosterol and sitostanol.

In addition to previous reports dietary plant stanol ester reduced tissue levels of plant sterols not only in plasma and liver but also in the brain with recorded increases in levels of campestanol and sitostanol ${ }^{26,33}$. However, the plasma, liver and brain levels of campestanol and sitostanol in Watanabe rabbits fed with RSO stanol ester-added diet still remained at low levels compared to campesterol and sitosterol in the rabbits fed with RSO sterol esters.

Feeding cholesterol and RSO plant sterol or stanol ester to heterozygous and homozygous Watanabe rabbits had no influence on cholesterol synthesis in brain. In heterozygous rabbits only we found a lower cholesterol content in the RSO sterol and stanol ester-fed rabbits. In contrast, in plant stanol esterfed homozygous rabbits cholesterol content in brain was not significantly but about $16 \%$ higher compared with standard diet-fed animals. However, we are unable to provide a valid explanation for this. It was previously demonstrated that an increased dietary intake of cholesterol in New Zealand white rabbits was associated with suppression of cholesterol synthesis in many extrahepatic tissues, but not in brain ${ }^{34}$. Additonally brain cholesterol content did not change either. Increased levels of plant sterols in brain of ABCG5/8 KO mice also showed no influence on cholesterol synthesis ${ }^{12}$. In sitosterolemic patients with increased plasma and brain non-cholesterol concentrations, brain cholesterol content did not differ from that of control subjects ${ }^{16}$. This confirms strict regulation of cholesterol metabolism in brain.

In homozygous Watanabe rabbits, RSO plant sterols and stanols had no influence on plasma cholesterol concentration, while in the cholesterol-fed heterozygous rabbits, supplementation of the diet with plant sterol or stanol esters decreased plasma cholesterol levels when compared to the animals receiving the diet with added cholesterol but no plant sterol or stanol esters. Plant sterols and stanols are noted for reducing cholesterol absorption by displacing cholesterol from mixed micelles ${ }^{35,36}$. In the present study, homozygous rabbits were not fed with cholesterol. Hence, no effect on dietary cholesterol absorption of plant sterols and stanols in the rabbits could be expected. Furthermore the extraction of biliary cholesterol may be impaired in homozygous Watanabe rabbits, leading to a situation where very limited amounts of cholesterol enters the digestive tract daily.

Cholesterol synthesis marker, the lathosterol:cholesterol ratio, in plasma, decreased in both homozygous and heterozygous rabbits, independent of the diet. In heterozygous rabbits this was caused by an increase of absolute amounts of cholesterol, though not significantly, in the plant stanol and sterol ester group (Table 2). In the homozygous animals absolute cholesterol levels remained unchanged while lathosterol itself decreased (Table 5). Thus the lathosterol:cholesterol ratio also decreased significantly in each group. As shown in a previous study, endogenous cholesterol synthesis decreased with aging in homozygous Watanabe animals ${ }^{37}$. This was in agreement with the results in homozygous but not with heterozygous animals in the present study. Unfortunately, we do not have a comparison in heterozygous animals which did not receive cholesterol. Thus, this biochemical marker does not provide an answer to the question whether feeding of cholesterol, RSO plant sterols or stanols has an effect on cholesterol synthesis in these rabbits.

The results with these novel RSO sterol and stanol ester preparations are valid for the Watanabe rabbit model used but whether the use of commercial food with added plant sterols and stanols also cause a similar elevation or reduction of total phytosterols in the brain tissue of man remains to be 
elucidated. To clarify the mechanisms by which plant sterols and stanols are transported into the brain and whether they have an influence on brain function(s) requires further investigation.

\section{Acknowledgements}

This work was completed on behalf of the NOFORISK Consortium and funded under the EU 6th Framework Food Quality and Safety Programme, project number FP6-506387. The authors thank Silvia Friedrichs, Meryem Gültekin, Merete Lykkegaard, Karen Roswall and the animal technicans for technical assistance.

\section{References}

1. Devaraj S \& Jialal I (2006) The role of dietary supplementation with plant sterols and stanols in the prevention of cardiovascular disease. Nutr Rev 64, 348-354.

2. Katan MB, Grundy SM, Jones P, Law M, Miettinen $T$ \& Paoletti R (2003) Efficacy and safety of plant stanols and sterols in the management of blood cholesterol levels. Mayo Clin Proc 78, 965-978.

3. Plat J \& Mensink RP (2005) Plant stanol and sterol esters in the control of blood cholesterol levels: mechanism and safety aspects. Am J Cardiol 96, 15D-22D.

4. Grundy SM, Cleeman JI, Merz CN, Brewer HB Jr, Clark LT, Hunninghake DB, Pasternak RC, Smith SC Jr \& Stone NJ (2004) Implications of recent clinical trials for the National Cholesterol Education Program Adult Treatment Panel III guidelines. Circulation 110, 227-239.

5. Watanabe Y, Ito T \& Shiomi M (1985) The effect of selective breeding on the development of coronary atherosclerosis in WHHL rabbits. An animal model for familial hypercholesterolemia. Atherosclerosis 56, 71-79.

6. Kita T, Brown MS, Watanabe Y \& Goldstein JL (1981) Deficiency of low density lipoprotein receptors in liver and adrenal gland of the WHHL rabbit, an animal model of familial hypercholesterolemia. Proc Natl Acad Sci U S A 78, $2268-2272$.

7. Watanabe Y (1980) Serial inbreeding of rabbits with hereditary hyperlipidemia (WHHL-rabbit). Atherosclerosis 36, 261-268.

8. NOFORISK (2007) Quantitative risk assessment strategies for novel foods. http://www.NOFORISK.org.

9. Dietschy JM \& Turley SD (2004) Thematic review series: brain Lipids. Cholesterol metabolism in the central nervous system during early development and in the mature animal. J Lipid Res 45, 1375-1397.

10. Lütjohann D, Brzezinka A, Barth E, Abramowski D, Staufenbiel M, von Bergmann K, Beyreuther K, Multhaup G \& Bayer TA (2002) Profile of cholesterol-related sterols in aged amyloid precursor protein transgenic mouse brain. J Lipid Res 43, $1078-1085$.

11. Yu L, von Bergmann K, Lütjohann D, Hobbs HH \& Cohen JC (2004) Selective sterol accumulation in ABCG5/ABCG8deficient mice. J Lipid Res 45, 301-307.

12. Jansen PJ, Lütjohann D, Abildayeva K, et al. (2006) Dietary plant sterols accumulate in the brain. Biochim Biophys Acta 1761, 445-453.

13. Thelen KM, Rentsch KM, Gutteck U, Heverin M, Olin M, Andersson U, von Eckardstein A, Björkhem I \& Lütjohann D (2006) Brain cholesterol synthesis in mice is affected by high dose of simvastatin but not of pravastatin. J Pharmacol Exp Ther 316, 1146-1152.
14. Heverin M, Bogdanovic N, Lütjohann D, Bayer T, Pikuleva I, Bretillon L, Diczfalusy U, Winblad B \& Björkhem I (2004) Changes in the levels of cerebral and extracerebral sterols in the brain of patients with Alzheimer's disease. J Lipid Res 45, $186-193$.

15. Salen G, Kwiterovich PO Jr, Shefer S, Tint GS, Horak I, Shore V, Dayal B \& Horak E (1985) Increased plasma cholestanol and 5 alpha-saturated plant sterol derivatives in subjects with sitosterolemia and xanthomatosis. J Lipid Res 26, 203-209.

16. Salen G, Horak I, Rothkopf M, et al. (1985) Lethal atherosclerosis associated with abnormal plasma and tissue sterol composition in sitosterolemia with xanthomatosis. J Lipid Res 26, 1126-1133.

17. Lütjohann D, Stroick M, Bertsch T, et al. (2004) High doses of simvastatin, pravastatin, and cholesterol reduce brain cholesterol synthesis in guinea pigs. Steroids 69, 431-438.

18. Dorszewska J \& Adamczewska-Goncerzewicz Z (2000) Patterns of free and esterified sterol fractions of the cerebral white matter in severe and moderate experimental hypoxia. Med Sci Monit 6, $227-231$.

19. Kritchevsky D \& Defendi V (1962) Deposition of tritium labelled sterols (cholesterol, sitosterol, lanosterol) in brain and other organs of the growing chicken. J Neurochem 9, 421-425.

20. Prasad VV, el-Maraghy E, Ponticorvo L \& Lieberman S (1985) Detection in extracts of bovine brain of lipophilic complexes of sulfate esters of cholesterol and beta-sitosterol. Proc Natl Acad Sci U S A 82, 2657-2659.

21. Ratnayake WM, Plouffe L, Hollywood R, L'Abbe MR, Hidiroglou N, Sarwar G \& Mueller R (2000) Influence of sources of dietary oils on the life span of stroke-prone spontaneously hypertensive rats. Lipids 35, 409-420.

22. Bhattacharyya AK \& Connor WE (1974) Beta-sitosterolemia and xanthomatosis. A newly described lipid storage disease in two sisters. J Clin Invest 53, 1033-1043.

23. Lütjohann D \& von Bergmann K (1997) Phytosterolaemia: diagnosis, characterization and therapeutical approaches. Ann Med 29, 181-184.

24. Yu L, Hammer RE, Li-Hawkins J, Von Bergmann K, Lütjohann D, Cohen JC \& Hobbs HH (2002) Disruption of Abcg5 and Abcg8 in mice reveals their crucial role in biliary cholesterol secretion. Proc Natl Acad Sci U S A 99, 16237-16242.

25. Lütjohann D, Björkhem I, Beil UF \& von Bergmann K (1995) Sterol absorption and sterol balance in phytosterolemia evaluated by deuterium-labeled sterols: effect of sitostanol treatment. J Lipid Res 36, 1763-1773.

26. Gylling H, Puska P, Vartiainen E \& Miettinen TA (1999) Serum sterols during stanol ester feeding in a mildly hypercholesterolemic population. J Lipid Res 40, 593-600.

27. Jones PJ, Raeini-Sarjaz M, Ntanios FY, Vanstone CA, Feng JY \& Parsons WE (2000) Modulation of plasma lipid levels and cholesterol kinetics by phytosterol versus phytostanol esters. J Lipid Res 41, 697-705.

28. Sudhop T, Sahin Y, Lindenthal B, Hahn C, Luers C, Berthold HK \& von Bergmann K (2002) Comparison of the hepatic clearances of campesterol, sitosterol, and cholesterol in healthy subjects suggests that efflux transporters controlling intestinal sterol absorption also regulate biliary secretion. Gut 51, 860-863.

29. Teunissen CE, De Vente J, von Bergmann K, Bosma H, van Boxtel MP, De Bruijn C, Jolles J, Steinbusch HW \& Lütjohann D (2003) Serum cholesterol, precursors and metabolites and cognitive performance in an aging population. Neurobiol Aging 24, 147-155.

30. Ketomaki A, Gylling H \& Miettinen TA (2005) Non-cholesterol sterols in serum, lipoproteins, and red cells in statin-treated FH subjects off and on plant stanol and sterol ester spreads. Clin Chim Acta 353, 75-86.

31. Rall CJ, Hoeg JM, Gregg RE, Law SW, Monge JC, Meng MS, Zech LA \& Brewer HB Jr (1988) Enhanced apolipoprotein 
E production with normal hepatic mRNA levels in the Watanabe heritable hyperlipidemic rabbit. Arteriosclerosis 8, 804-809.

32. Thelen KM, Falkai P, Bayer TA \& Lütjohann D (2006) Cholesterol synthesis rate in human hippocampus declines with aging. Neurosci Lett 403, 15-19.

33. Miettinen TA, Puska P, Gylling H, Vanhanen H \& Vartiainen E (1995) Reduction of serum cholesterol with sitostanol-ester margarine in a mildly hypercholesterolemic population. $N$ Engl $J$ Med 333, 1308-1312.

34. Andersen JM, Turley SD \& Dietschy JM (1982) Relative rates of sterol synthesis in the liver and various extrahepatic tissues of normal and cholesterol-fed rabbits. Relationship to plasma lipoprotein and tissue cholesterol levels. Biochim Biophys Acta 711, 421-430.

35. Ikeda I, Tanabe Y \& Sugano M (1989) Effects of sitosterol and sitostanol on micellar solubility of cholesterol. J Nutr Sci Vitaminol (Tokyo) 35, 361-369.

36. Mel'nikov SM, Seijen ten Hoorn JW \& Eijkelenboom AP (2004) Effect of phytosterols and phytostanols on the solubilization of cholesterol by dietary mixed micelles: an in vitro study. Chem Phys Lipids 127, 121-141.

37. Dietschy JM, Kita T, Suckling KE, Goldstein JL \& Brown MS (1983) Cholesterol synthesis in vivo and in vitro in the WHHL rabbit, an animal with defective low density lipoprotein receptors. J Lipid Res 24, 469-480. 